Pacific Journal of Mathematics

A PROBLEM IN COMPACT LIE GROUPS AND FRAMED 


\title{
A PROBLEM IN COMPACT LIE GROUPS AND FRAMED COBORDISM
}

\author{
Hillel H. Gershenson
}

\begin{abstract}
Given a compact, connected, $k$-dimensional, oriented Lie group $G$ or a faithful orthogonal representation $T$ of such a $G$ there arises an element of the $k$ th framed cobordism group $\Omega_{k}^{f r}$. The study of these elements is begun, and some algebraic properties of the situation are discussed. The remaining problem is to relate such properties of the elements in $\Omega_{k}^{f r}$ as order or Adams $d$ and $e$ invariants to Lie theory.
\end{abstract}

If $G$ is a $k$-dimensional Lie group its tangent bundle may be trivialized by choosing a linear isomorphism of its Lie algebra $\mathscr{L}(G)$ with Euclidean space $R^{k}$, and using right multiplication to give an isomorphism of the tangent space at any point with the tangent space at the identity which is, of course, the Lie algebra. If $G$ is compact and oriented every trivialization of the tangent bundle gives rise to a trivialization of the stable normal bundle (see the discussion of tangential and normal structures on p. 23 of [2]) and hence to an element of the $k$ th framed cobordism group $\Omega_{k}^{f r}$. If two choices of linear isomorphisms of $\mathscr{L}(G)$ with $R^{k}$ differ by an element of $G L_{k}(R)$ of positive determinant it is easily seen that the corresponding tangential trivializations are homotopic through trivializations and hence determine the same element of $\Omega_{k}^{f r}$. Thus, a compact, oriented $k$-dimensional Lie group $G$ gives rise to a well-defined element $[G] \in \Omega_{k}^{f r}$.

Now assume in addition that $G$ is connected and let $T: G \rightarrow \operatorname{SO}(n)$ be a faithful representation of $G$. $T$ embeds $G$ in Euclidean $n^{2}$-space. If $G$ is $k$-dimensional then $k \leqq n(n-1) / 2<n^{2} / 2$, since $\operatorname{dim} G \leqq$ $\operatorname{dim} \mathrm{SO}(n)$, so that codim $G>k$ and the normal bundle of $G$ in this embedding is already stable. We shall always assume a fixed orientation in any Euclidean space we discuss; in particular view Euclidean $n^{2}$-space as $M(n)$, the space of $n \times n$ real matrices and choose an orthonormal basis $e_{i j}, 1 \leqq i, j \leqq n$, where $e_{i j}$ is the matrix with one in the ijth position and zeroes elsewhere. Orient $M(n)$ by putting the $e_{i j}$ in lexicographic order, so that the ordered basis is $e_{11}, e_{12}, \cdots$, $e_{1 n}, e_{21}, \cdots, e_{2 n}, \cdots, e_{n 1}, \cdots, e_{n n}$. Make the convention that $M(n)$ is always oriented this way, and also assume that the matrix of any linear transformation from $M(n)$ to itself is always written with respect to this ordered basis.

Returning to the faithful representation $T$, choose an orthonormal basis $\tau_{1}, \cdots, \tau_{k}$ for $\tau_{I}$ the tangent $k$-plane to $T(G)$ at the identity $I$. 
Then choose an orthonormal basis $\nu_{1}, \cdots, \nu_{n^{2}-k}$ for $\tau_{I}^{1}$ in such a way that the basis $\tau_{1}, \cdots, \tau_{k}, \nu_{1}, \cdots, \nu_{n^{2}-k}$ determines the fixed orientation of $M(n)$.

Since $G$ (identified with $T(G)$ ) is contained in $\mathrm{SO}(n)$, right multiplication of elements of $M(n)$ by an element $g \in G$ defines an element $\rho_{g}$ in $\mathrm{SO}\left(n^{2}\right)$. Since $\rho_{g \mid G}$ is a diffeomorphism of $G$ and $d \rho_{g}$, the differential of $\rho_{g}$ (viewed as a map $\rho_{g}: M(n) \rightarrow M(n)$ ) is a constant element of $\mathrm{SO}\left(n^{2}\right), d \rho_{g}$ maps the tangent (resp. normal) space at $I$ to the tangent (resp. normal) space at $g$. Define $\nu_{i}(g)=d \rho_{g}\left(\nu_{i}\right)$, and observe that this defines a framing of the stable normal bundle of $G, \nu(G)$, and thus an element $[T] \in \Omega_{f r}^{k}$. Observe also that $\tau_{j}(g)=$ $d \rho_{g}\left(\tau_{j}\right)$ defines a framing of $\tau(G)$ which coincides with the framing discussed above in connection with the element [G].

The object of this note is to begin the study of the elements [G] and [T]. It is first shown that the element [T] does not depend on the choice of framing at $I$, and the question of the dependence of $[T]$ on the equivalence class of $T$ as representation is then discussed. The algebraic properties of the map $T \mapsto[T]$ are considered. The following statements are proved by example:

(A) If $S$ and $T$ are two faithful representations of $G,[S+T] \neq$ $[S]+[T]$ in general.

(B) If $T+m$ is the representation obtained by adding $m$ trivial representations to $T$, then in general $[T+m] \neq[T]$.

Some positive statements may be made, however,

Proposition 1. If $T_{1}: G_{1} \rightarrow \mathrm{SO}\left(n_{1}\right)$ and $T_{2}: G_{2} \rightarrow \mathrm{SO}\left(n_{2}\right)$ are two faithful representations of two compact, connected, oriented Lie groups of dimensions $k_{1}$ and $k_{2}$ respectively, and $T_{1} \oplus T_{2}: G_{1} \times G_{2} \rightarrow \operatorname{SO}\left(n_{1}+n_{2}\right)$ is their exterior direct sum, then $\left[T_{1} \oplus T_{2}\right]=(-1)^{e}\left[T_{1}+n_{2}\right]\left[T_{2}+n_{1}\right]$, where $e=k_{2}\left(n_{1}+k_{1}+n_{1} n_{2}\right)$ and the product on the right is taken in the framed cobordism ring $\Omega_{*}^{f r}$.

Proposition 2. Let $T: G \rightarrow \mathrm{SO}(n)$ be a faithful representation of a compact, connected k-dimensional Lie group.

(a) The sequence $[T],[T+1], \cdots,[T+m], \cdots$ of elements of $\Omega_{k}^{f r}$ is cyclic in $m$.

(b) The element $[G]$ occurs in the sequence of (a).

(c) If $r, s$ are positive integers then $[r T+s]$ occurs in the sequence of (a); in fact, $[r T+s]=\left[T+\left(r^{2}-1\right) n+s\right]$.

The problems that remain open may be summarized as: How do the Lie-theoretic properties of $G$ and $T$ affect the elements $[G]$ and [T]? We list two such problems: What conditions on $G$ and $T$ allow one to conclude that $[G]=0$ or $[T]=0$ ? Express the numbers that 
occur-such numbers as the orders of $[G]$ and $[T]$, the primes that occur in those orders, the Adams $d$ and $e$ invariants of $[G]$ and $[T]$, the length of the cycle of Proposition (2c) in terms of Lie theory.

Before proceeding to the proofs of the statements above, it is useful to make the matrix form of $d \rho_{g}$ explicit. Since $\rho_{g}$ is a linear map of $M(n)$ to itself, $d \rho_{g}$ is constant and $d \rho_{g}=\rho_{g}$. If $x=\left(x_{i j}\right) \in M(n)$ $g=\left(g_{k 1}\right) \in T(G)$, then $\rho_{g}(x)=\left(y_{s t}\right)$, where $y_{s t}=\sum_{q} x_{s q} g_{q t}$. Setting $x=$ $e_{i j}$ and looking at the formula proves Lemma 1 .

LeMma 1. As a matrix in $\mathrm{SO}\left(n^{2}\right), d \rho_{g}$ is obtained by stringing $n$ copies of $g^{t}=g^{-1}$ down the diagonal.

Lemma 2. In $\Omega_{k}^{f r},[T]$ is independent of choice of basis $\tau_{1}, \cdots$, $\tau_{k}\left(\right.$ resp. $\left.\nu_{1}, \cdots, \nu_{n^{2}-k}\right)$ of tangent (resp. normal) space at the identity.

Proof. Let $\tau_{1}^{\prime}, \cdots, \tau_{k}^{\prime}, \nu_{1}^{\prime}, \cdots, \nu_{n^{2}-k}^{\prime}$ be another choice of orthonormal, properly oriented bases of $\tau_{I}$ and $\tau_{I}^{\perp}=\nu_{I}$ respectively. Define $L \in \operatorname{SO}\left(n^{2}\right)$ by $L\left(\tau_{i}\right)=\tau_{i}^{\prime}$ and $L\left(\nu_{j}\right)=\nu_{j}^{\prime} . \quad L$ is in that subgroup $H$ of $\mathrm{SO}\left(n^{2}\right)$ which keeps $\tau_{I}$ and $\nu_{I}$ invariant, so that $H$ is isomorphic to $\mathrm{SO}(k) \times \mathrm{SO}\left(n^{2}-k\right)$. Let $\sigma$ be a smooth path in $H$ from $L$ to the identity. Then, for $0 \leqq t \leqq 1$ and $g \in T(G), \nu_{j}(g)_{t}=\left(d \rho_{g}\right)\left(\sigma(t)\left(\nu_{j}\right)\right)$ defines a homotopy of one normal framing to the other through normal framings, so the two framings represent the same element of $\Omega_{k}^{f r}$ (see, e.g., p. 190 of [1]).

Note that it is important to maintain orientation; if the orientation is changed the sign of [T] changes. It is also easy to see that if $G$ is not connected but has, say, $q>1$ components, and if each component $G_{\alpha}$ is identified with $G_{0}$, the component of the identity, by right multiplication by a fixed $g_{\alpha}^{-1}, g_{\alpha} \in G_{\alpha}$, and if $T: G \rightarrow O(n)$ is a faithful representation and $T_{0}=T \mid G_{0}$, then $\nu(G)$ may be framed as before, and in this case $[T]=q\left[T_{0}\right]$. The nonconnected case will not be explicitly considered further, although much of the discussion below remains valid in that case.

Definition. Let $S$ and $T$ be two (not necessarily faithful) orthogonal representations of the group $G . S$ and $T$ are centrally equivalent means:

(i) $S$ and $T$ have the same rank $n$.

(ii) There exists $A \in \mathrm{O}(n)$ such that $S=A T A^{-1}$.

(iii) There exists $B \in \mathrm{O}(n)$ such that $\operatorname{det} A=\operatorname{det} B$ and $B$ centralizes $T(G)$, i.e., $B T(g)=T(g) B$ for all $g \in G$.

We state some obvious observations as a lemma. 
Lemma 3. (i) Central equivalence is an equivalence relation which implies the usual equivalence relation.

(ii) If the rank is odd, central equivalence coincides with the usual equivalence relation.

(iii) If $S$ and $T$ are centrally equivalent, so are $S+1$ and $T+$ 1; if $S$ and $T$ are equivalent, $S+1$ and $T+1$ are centrally equivalent.

(iv) If $S$ and $T$ are two representations of $G, S+T$ and $T+S$ are centrally equivalent.

(v) If $S$ and $T$ are equivalent unitary representions of $G$, their "realifications" are centrally equivalent.

Proposition 3. (i) If $S$ and $T$ are centrally equivalent faithful representations of the compact, oriented Lie group $G$, then $[S]=[T]$.

(ii) If $S$ and $T$ are two faithful orthogonal representations of $G,[S+T]=[T+S]$.

(iii) If $S$ and $T$ are equivalent faithful unitary representations of $G$, then $[S]=[T]$.

Proof. Let $A, B \in \mathrm{O}(n)$ be such that $S=A T A^{-1}$, $\operatorname{det} A=\operatorname{det} B$, and $B$ centralizes $T(G)$. Let $\sigma: I \rightarrow \mathrm{O}(n)$ be a smooth path from $B$ to $A$. Let $T_{t}: G \rightarrow \mathrm{SO}(n)$ be defined by $T_{t}=\sigma(t) T \sigma(t)^{-1}$, and let $\hat{\sigma}: M(n) \rightarrow M(n)$ be defined by $\hat{\sigma}_{t}(M)=\sigma(t) M \sigma(t)^{-1}$. Observe that for every $t \in I, \hat{\sigma} \in \mathrm{SO}\left(n^{2}\right)$. Choose a tubular neighborhood $\nu$ of $T(G)$ and, if $x \in \nu$, set $x_{t}=\hat{\sigma}_{t} B^{-1} x B$. Note that $x_{0}=x$, and $T_{0}(g)=T(g)$ for all $g \in G$. Choose a basis for the normal bundle of $T(G)$ at the identity following the orientation convention described above. Then, for $t \in I$ and $g \in G$, framing $T_{t}(G)$ by applying $d T_{t}(g)$ to the basis chosen above defines an isotopy of framed manifolds. Since $T_{1}=S$ and Lemma 2 shows that $[S]$ can be defined using the framing defined here, it follows that $[S]=[T]$. This proves (i).

The other two parts follow from (i) and Lemma 3.

A slight extension of these ideas may be useful in considering tensor products. If $T: G \rightarrow \mathrm{SO}(n)$ is a representation with finite kernel of a compact, connected, oriented Lie group, then $T$ induces a faithful representation $T^{\prime}$ of $G^{\prime}=G / \operatorname{ker} T$, where $G^{\prime}$ is again a compact connected Lie group which inherits an orientation for $G$. We may define $[T]=\left[T^{\prime}\right]$. If $G$ is semi-simple in addition, and $S$ and $T$ are two representations of $G$ which have finite kernel, then so does $S \otimes T$, although $S \otimes T$ need not be faithful even if $S$ and $T$ are. Thus, $[S \otimes T]$ can be defined, and it can be shown as in the proof of Lemma 4 that $[S \otimes T]=[T \otimes S]$. If $S$ and $T$ are faithful representations 
of $G$ (or representations with finite kernel) it would be of interest to obtain general formulas for $[S+T]$ and, if $G$ is semi-simple, $[S \otimes T]$, in terms of $[S]$ and $[T]$.

Proof of Proposition 1. Suppose $T: G \rightarrow \mathrm{SO}(n)$ is a faithful representation, and consider $T+m: G \rightarrow \mathrm{SO}(n+m)$. Linearly embed $M(n)$ in $M(n+m)$ by the map $\theta: e_{i j} \rightarrow e_{i j}$. Then, if $\tau_{1}, \cdots, \tau_{k}$ and $\nu_{1}, \cdots, \nu_{n^{2}-k}$ are properly oriented frames in $\tau_{I}$ and $\nu_{I}$ respectively, $\theta\left(\tau_{1}\right), \cdots, \theta\left(\tau_{k}\right)$ is a tangential frame of $(T+m)(G)$ at the identity, and $\theta\left(\nu_{1}\right), \cdots, \theta\left(\nu_{n^{2}-k}\right), e_{r s}, r>n$ or $s>n$, with the $e_{r s}$ in lexicographic order, is a normal frame at the identity of $(T+m)(G)$. These frames do not, in general, give the proper orientation to the normal frame according to our conventions; a computation shows that this orientation is $(-1)^{f}$ times the proper orientation, where $f=1 / 2 m n^{2}(n-1)$. Thus, normally framing $(T+m)(G)$ by moving the given frame at $I$ by $d \rho_{g}$ for each $g \in G$ gives a framed manifold representing $(-1)^{f}$ $[T+m]$. Applying Lemma 1 to the representation $T+m$ shows that, if $g \in G, d \rho_{g}$ fixes $e_{r s}$ whenever $s>n$. Hence, if $V \subset M(n+m)$ is the $m(n+m)$ dimensional subspace of $M(n+m)$ spanned by the $e_{r s}, s>n, G$ may be viewed as embedded in $V^{\perp}$ by orthogonal projection of $(T+m) G$ into $V^{\perp}$, and the normal frame at $g$ may be taken as $d \rho_{g}$ applied to $\theta\left(\nu_{1}\right), \cdots, \theta\left(\nu_{n^{2}-k}\right), e_{r s}, r>n, s<n$, in lexicographic order, all of which vectors lie in $V^{\perp}$. Identifying $V^{\llcorner}$with Euclidean $n(n+m)$ space shows that $G$ is now embedded in $R^{n(n+m)}$ as a framed manifold, and that, with this framing, $G$ represents $(-1)^{f}[T+m]$.

Now consider the situation of the proposition. Let $\tau_{1}, \cdots, \tau_{k_{1}}$ and $\tau_{1}^{\prime}, \cdots, \tau_{k_{2}}^{\prime}$ be tangential frames at the identity for $T_{1}\left(G_{1}\right)$ and $T_{2}\left(G_{2}\right)$ respectively, and let corresponding normal frames be $\nu_{1}, \cdots, \nu_{n_{1}^{2}-k_{1}}$ and $\nu_{1}^{\prime}, \cdots, \nu_{n_{2}^{2}-k_{2}}^{\prime}$. Define $\theta: M\left(n_{1}\right) \rightarrow M\left(n_{1}+n_{2}\right)$ by $\theta\left(e_{i j}\right)=e_{i j}$, and $\theta^{\prime}: M\left(n_{2}\right) \rightarrow M\left(n_{1}+n_{2}\right)$ by $\theta^{\prime}\left(e_{s t}\right)=e_{n_{1}+s, n_{1}+t}$. A tangential frame for $\left(T_{1} \oplus T_{2}\right)\left(G_{1} \times G_{2}\right)$ at the identity is given by $\theta\left(\tau_{1}\right), \cdots, \theta\left(\tau_{k_{1}}\right), \theta^{\prime}\left(\tau_{1}^{\prime}\right), \cdots$, $\theta^{\prime}\left(\tau_{k_{2}}^{\prime}\right)$, and a normal frame by $\theta\left(\nu_{1}\right), \cdots, \theta\left(\nu_{n_{1}^{2}-k_{1}}\right), e_{r s}, \theta^{\prime}\left(\nu_{1}^{\prime}\right), \cdots$, $\theta^{\prime}\left(\nu_{n_{2}^{2}-k_{2}}^{\prime}\right), e_{t u}$, where $n_{1}+1 \leqq r \leqq n_{1}+n_{2}, 1 \leqq s \leqq n_{1}, 1 \leqq t \leqq n_{1}, n_{1}+$ $1 \leqq u \leqq n_{1}+n_{2}$ and the $e_{r s}$ and $e_{t u}$ are taken in lexicographic order. View $M\left(n_{1}+n_{2}\right)$ as the direct sum of two Euclidean spaces $V$ and $W, V$ of dimension $n_{1}\left(n_{1}+n_{2}\right)$ and spanned by the $e_{i j}$ with $1 \leqq j \leqq$ $n_{1}$, and $W$ of dimension $n_{2}\left(n_{1}+n_{2}\right)$ and spanned by the $e_{i j}$ with $n_{1}+$ $1 \leqq j \leqq n_{1}+n_{2}$. Apply Lemma 1 to the case of $T_{1} \oplus T_{2}$, and observe that the action of $d \rho\left(g_{1}, g_{2}\right)$ for any $\left(g_{1}, g_{2}\right) \in G_{1} \times G_{2}$ gives rise to the framing discussed above in the case of $T_{1}+n_{2}$ when applied to $\theta\left(\nu_{1}\right), \cdots, \theta\left(\nu_{n_{1}^{2}-k_{1}}\right), e_{r s} ; n_{1}+1 \leqq r \leqq n_{1}+n_{2}, 1 \leqq s \leqq n_{1}$, where $G_{1}$ is viewed as embedded in $V$, and similarly gives rise to the framing discussed above in the case of $T_{2}+n_{1}$ when $G_{2}$ is viewed as embedded in $W$. Therefore, the framing on $\left(T_{1} \oplus T_{2}\right)\left(G_{1} \times G_{2}\right)$ represents, on 
the one hand, $(-1)^{g}\left[T_{1}+n_{2}\right]\left[T_{2}+n_{1}\right], g=1 / 2 n_{1} n_{2}\left(n_{1}^{2}+n_{2}^{2}-n_{1}-n_{2}\right)$, and on the other $\varepsilon\left[T_{1} \oplus T_{2}\right]$, where $\varepsilon= \pm 1$. This sign is positive if the orientation of the normal frame at the identity coincides with the one determined by our orientation convention, and negative otherwise. An exercise in changing bases and computing the signs of permutations then shows that $\varepsilon=(-1)^{h}$, where $h=n_{1} n_{2}\left(n_{2}^{2}+n_{1} n_{2}\right)+$ $k_{2}\left(n_{1}^{2}-k_{1}\right)+k_{2} n_{1} n_{2}+g$, and the proposition follows.

CoROLLARY 1. $\left[T_{1} \oplus T_{2}\right]=(-1)^{m}\left[T_{2} \oplus T_{1}\right]$, where $m=k_{1} n_{2}\left(n_{1}+1\right)+$ $k_{2} n_{1}\left(n_{2}+1\right)$.

Before proving Proposition 2 some discussion and a lemma are needed. Suppose $M^{k}$ is a $k$-dimensional stably parallelizable compact manifold embedded in $R^{N}$ with stable normal bundle $\nu^{N-k}$ and that $\varphi$ and $\psi$ are trivializations of $\nu^{N-k}$. In [1] a difference element $d(\varphi, \psi)$ in $K^{-1}(M)$ (real $K$-theory) was defined. Let $T: G \rightarrow \mathrm{SO}(n)$ be a faithful representation of a compact, connected, oriented Lie group. The tangential trivialization of $G$ discussed above gives rise [2] to a unique (in the sense of equivalence of trivializations [1] [2]) trivialization of the normal bundle of $G$ for any embedding of $G$ in a Euclidean space of sufficiently high dimension. In particular, $n^{2}$ and $2 n^{2}$ are sufficiently high dimensions. Call this trivialization $\widetilde{G}$. The representation $T$ also gives rise to a trivialization of the normal bundle, as has been described. Call this trivialization $\widetilde{T}$. As a map of $G$ into $\mathrm{SO}(n) \subset 0, T$ represents an element of $K^{-1}(G)$, which we call $\langle T\rangle$.

\section{Lemma 4. In $K^{-1}(G), d(\widetilde{G}, \widetilde{T})=n\langle T\rangle$.}

Proof. The tangential framing of $G$ is independent of $T$, and hence $\widetilde{G}$, while it may be described in terms of $T$, will be independent of $T$ as trivialization of the normal bundle (see the discussion on $p$. 23 of [2] for the relationship between tangential and normal structures). The trivialization of the normal bundle is obtained as follows. If $M$ is a compact, $k$-dimensional, stably parallelizable manifold embedded in $R^{N} \subset R^{N+1}$, where $N$ is sufficiently large, let $\tau_{1}(x), \cdots, \tau_{k+1}(x)$ be a trivialization of $\tau(M) \oplus 1$, where at each point $x \in M$ this set of vectors is an orthonormal basis for $\tau_{x} \oplus R$ (a Riemannian structure on $M$ is assumed). Then a trivialization $\nu_{1}(x), \cdots, \nu_{N-k}(x)$ of the normal bundle of $M$ corresponds to the given tangential trivialization precisely when the map $x \mapsto\left(\tau_{1}(x), \cdots, \tau_{k+1}(x), \nu_{1}(x), \cdots, \nu_{N-k}(x)\right)$, which is a map of $M$ into $\mathrm{O}(N+1) \subset O$ represents zero in $K^{-1}(M)$, i.e., is null homotopic. If $M$ is parallelizable, the same thing may be done in $R^{N}$ with $k$ tangent vector fields.

In our case such a framing of the normal bundle may be obtained 
by viewing Euclidean $2 n^{2}$-space as $M(n) \oplus M(n)$, and embedding $M(n)$ in it as the first summand. This embeds $T(G)$ in $M(n) \oplus M(n)$. Again, choose a tangential framing $\tau_{1}, \cdots, \tau_{k}$ and a normal framing $\nu_{1}, \cdots$, $\nu_{n^{2}-k}$ at the identity of $T(G)$ in $M(n)$. In $M(n) \oplus M(n)$ a tangential framing at the identity is then given by $\left(\tau_{1}, 0\right), \cdots,\left(\tau_{k}, 0\right)$ and a normal one by $\left(\nu_{1}, 0\right), \cdots,\left(\nu_{n^{2}-k}, 0\right),\left(0, e_{i j}\right)$, the $e_{i j}$ being taken, say in lexicographic order. If these framings at the identity are then translated by the action of the elements

$$
A_{g}=\left(\begin{array}{cc}
d \rho_{g} & 0 \\
0 & \left(d \rho_{g}\right)^{t}
\end{array}\right) \text { in } \mathrm{SO}\left(2 n^{2}\right),
$$

tangential and normal framings of $T(G)$ are obtained which correspond to each other as described above. Hence the framing of the normal bundle is, as framing, independent of $T$.

If we translate the normal framing at the identity by the elements

$$
B_{g}=\left(\begin{array}{cc}
d \rho_{g} & 0 \\
0 & I
\end{array}\right) \text { in } \mathrm{SO}\left(2 n^{2}\right) \text { we }
$$

obtain a framing of $T(G)$ which represents [T]; it is just the framing already described stabilized to co-dimension $2 n^{2}-k$. Take the first framing of the normal bundle to be $\widetilde{G}$ and the second to be $\widetilde{T}$.

It follows from the definition of the difference element in [1] that $d(\widetilde{G}, \widetilde{T}) \in K^{-1}(G)$ is represented by the map $\delta: G \rightarrow \mathrm{SO}\left(2 n^{2}-k\right)$ to be described next.

Fix an ordered orthonormal basis of Euclidean $\left(2 n^{2}-k\right)$-space. The fixed normal frame at the identity then defines a norm preserving monomorphism $F: R^{2 n^{2}-k} \rightarrow M(n) \oplus M(n)$. Let $\pi: M(n) \oplus M(n) \rightarrow$ $R^{2 n^{2}-k}$ be orthogonal projection on the image of $F$ followed by $F^{-1}$. Then $\delta(g)=\pi A_{g} B_{g}^{t} F$. If $C_{g}=A_{g} B_{g}^{t} \in \mathrm{SO}\left(2 n^{2}\right)$, then

$$
C_{g}=\left(\begin{array}{cc}
I & 0 \\
0 & \left(d \rho_{g}\right)^{t}
\end{array}\right) \text {. }
$$

It follows from the definitions of the frame at the identity and $F$ that ker $\pi$ is contained in the first summand of $M(n) \oplus M(n)$. Since $C_{g}$ is the identity map when restricted to that summand and $C_{g}$ sends the second summand to itself, we see that $d(\widetilde{G}, \widetilde{T})$ is also represented by the map $g \mapsto\left(d \rho_{g}\right)^{t} \in \mathrm{SO}\left(n^{2}\right)$. Lemma 4 then follows from Lemma 1 .

Proof of Proposition 2. According to the theorem of [1], $[T]=$ $[G]+\varphi(\widetilde{G}) \widetilde{J}^{-1} d(\widetilde{G}, \widetilde{T})$, where $d(\widetilde{G}, \widetilde{T})$ is projected into $\widetilde{K}^{-1}(G), \widetilde{J}^{-1}$ is the reduced $J$-morphism mapping $\widetilde{K}^{-1}(G)$ to $\widetilde{S}^{0}(G)$, the reduced stable cohomotopy group of $G$ in degree zero (or the reduced cohomology group of $G$ with coefficients in the sphere spectrum), and $\varphi(\widetilde{G})$ is a map of $\widetilde{S}^{\prime}(G)$ to $Q_{k}^{f r}$ which depends only on $\widetilde{G}$ up to equivalence of 
framings. In $K^{-1}(G),\langle T+k\rangle=\langle T\rangle$. Then, by Lemma 4, $d(\widetilde{G}$, $(\widetilde{T+k)})=(n+k)\langle T+k\rangle=(n+k)\langle T\rangle$, so that $d(\widetilde{G},(\widetilde{m T+p}))=$ $(m n+p)\langle m T\rangle=m(m n+p)\langle T\rangle=\left(n+\left(m^{2}-1\right) n+m p\right)\langle T\rangle=$ $d\left(\widetilde{G},\left(T+\left(m^{2}-1\right) n+p\right)\right)$. Hence, $[m T+p]=\left[T+\left(m^{2}-1\right) n+p\right]$, which proves part (c).

Since $G$ is connected, $\widetilde{S}^{0}(G)$ is a finite commutative ring unber the product in $S^{*}(G)$. (Note that this is the first time that the hypothesis of connectivity is really necessary.) In [1] it is shown that $\widetilde{J}^{-1}: \widetilde{K}^{-1}(G) \rightarrow \widetilde{S}^{0}(G)$ is a homomorphism from $\widetilde{K}^{-1}(G)$ to a modified abelian group structure on $\widetilde{S}^{0}(G)$. Hence, $\widetilde{J}^{-1} d(\widetilde{G},(\widetilde{T+k}))=\widetilde{J}^{-1}((n+$ $k)\langle T\rangle)$ is the $(n+k)$-fold sum of an element in a finite abelian group with itself. Therefore, as $k$ changes, $\widetilde{J}^{-1} d(\widetilde{G},(\widetilde{T+k}))$ changes cyclically, and, for some $k, \widetilde{J}^{-1} d(\widetilde{G},(\widetilde{T+k}))=0$. This proves parts (a) and (b).

Now consider statements (A) and (B), which will be proved by example. The methods used will involve Lemma 4 and the results of [1] as used in the proof of Proposition 3. In the examples, $G$ will always be $S^{3}=\operatorname{Sp}(1)$ and $T_{0}$ will be the representation of $\operatorname{Sp}(1)$ in SO(4) called $\sigma$ on p. 116 of [3], which is defined by viewing $q \in$ $\mathrm{Sp}(1)$ as a unit quaternion, and $q^{\prime} \in R^{4}$ as a quaternion and setting $\sigma(q) q^{\prime}=q q^{\prime}$. This map defines an element called $\beta_{3} \in \pi_{3}(\mathrm{SO}(4))$ which yields a generator of $\pi_{3}(0)$ under the inclusion $\mathrm{SO}(4) \subset 0$ [3]. The image of $\beta_{3}$ under the $J$-homomorphism generates $\Omega_{3}^{f r}$, as is wellknown, and is in fact the generator usually called $\nu$. We shall not stop to prove this here since it is not necessary; for our purposes, we may define the generator $\nu \in \Omega_{3}^{f r} \approx Z_{24}$ as $\nu=J\left(\beta_{3}\right)$.

The proof of Proposition 1 of [1] shows that in the case where $G$ (or the manifold, not necessarily a group, which is considered in that proposition) is a sphere, the map which we have called $\varphi(\widetilde{G})$ in the case considered here is the identity (recall that $\widetilde{S}^{0}\left(S^{n}\right)=\Omega_{n}^{f r}$ ) if the orientations involved are correctly chosen. Further, in the case of a sphere both group structures on $\widetilde{S}^{0}\left(S^{n}\right)$ coincide, so that the formula used in the proof of Proposition 2 becomes

$$
[T]=[G]+\widetilde{J}^{-1} d(\widetilde{G}, \widetilde{T}),
$$

where $\widetilde{J}^{-1}: \widetilde{K}^{-1}\left(S^{3}\right) \rightarrow \Omega_{3}^{f r}$ is the usual $J$-homomorphism.

Lemma 4 applied to this case then says that $d\left(\widetilde{G},\left(\widetilde{T_{0}+k}\right)\right)=$ $(4+k)\left\langle T_{0}\right\rangle$. Since $\widetilde{J}^{-1}\left\langle T_{0}\right\rangle=\nu, \widetilde{J}^{-1} d\left(\widetilde{G},\left(\widetilde{T_{0}+k}\right)\right)=(4+k) \nu$, so $\left[T_{0}+k\right]=$ $[G]+(4+k) \nu$. Therefore, $\left[T_{0}+k\right]=\left[T_{0}\right]$ precisely when $k \nu=0$, or $k \equiv 0(\bmod 24)$. This proves statement $(B)$. Note that the length of the cycle of Proposition (2a) is twenty-four in this case, so that all of $\Omega_{3}^{f r}$ is filled up, and that $\left[T_{0}+k\right]=[G]$ precisely when $k \equiv$ $-4(\bmod 24)$. 
Proposition (2c) tells us that $\left[m T_{0}\right]=\left[T_{0}+4\left(m^{2}-1\right)\right]=[G]+$ $4 m^{2} \boldsymbol{\nu}$. Then, $\left[p T_{0}\right]=[G]+4 p^{2} \boldsymbol{\nu}$, and $\left[m T_{0}+p T_{0}\right]=\left[(m+p) T_{0}\right]=$ $[G]+4(m+p)^{2} \nu$. Therefore, $\left[m T_{0}\right]+\left[p T_{0}\right]=\left[m T_{0}+p T_{0}\right]$ precisely when $8 m p \equiv 0(\bmod 24)$ or $m p \equiv 0(\bmod 3)$. In particular, $\left[T_{0}\right]+\left[T_{0}\right] \neq$ $\left[T_{0}+T_{0}\right]$, which proves statement (A).

In these examples it should be pointed out that in fact $[G]=\nu$ if the orientation of $G$ is picked properly, and that this orientation can be picked so as to yield $[G]=\nu$ at the same time that $\varphi(\widetilde{G})$ is the identity. The formulas of the last paragraphs then become

$$
\begin{aligned}
{\left[T_{0}\right] } & =5 \nu \\
{\left[T_{0}+k\right] } & =(5+k) \nu \\
{\left[m T_{0}\right] } & =\left(4 m^{2}+1\right) \nu .
\end{aligned}
$$

Seeing that $[G]=\nu$ involves a very tedious matrix calculation which is not described in detail here. Briefly, by writing down the representation $T_{0}$ it is seen that $S^{3}$ is embedded in $R^{16} \subset R^{32}$ as the intersection of the 15-sphere of radius two with a certain 4-plane. Using this fact, a normal trivialization of $T_{0}(G)$ can be written down which represents zero in $\Omega_{3}^{f r}$. The difference element between this framing and $\widetilde{G}$ can then be written down and, by a suitable choice of inner automorphisms, recognized as representing the image of $\beta_{3}$ in $\pi_{3}(\mathrm{SO}(29))$, and therefore as representing a generator of $\widetilde{K}^{-1}\left(S^{3}\right)$. That $[G]=\nu$ then follows from an application of the theorem of [1].

\section{REFERENCES}

1. H. H. Gershenson, On framed cobordism classes representable on a fixed manifold, Math. Z., 122 (1971), 189-202.

2. N. Steenrod, The Topology of Fibre Bundles, Princeton University Press, 1951.

3. R. Stong, Notes on Cobordism Theory, Princeton University Press, 1968.

Received October 17, 1972. Supported by NSF Grant GP-34021.

University of Minnesota 



\section{PACIFIC JOURNAL OF MATHEMATICS}

\section{EDITORS}

RICHARD ARENS (Managing Editor)

University of California

Los Angeles, California 90024

R. A. BeAumont

University of Washington

Seattle, Washington 98105
J. DugundJI*

Department of Mathematics

University of Southern California

Los Angeles, California 90007

D. Gilbarg and J. Milgram

Stanford University

Stanford, California 94305

\section{ASSOCIATE EDITORS}

E. F. BECKENBACH

B. H. NEUMANN

F. WOLF

K. YoSHIDA

\section{SUPPORTING INSTITUTIONS}

UNIVERSITY OF BRITISH COLUMBIA
CALIFORNIA INSTITUTE OF TECHNOLOGY
UNIVERSITY OF CALIFORNIA
MONTANA STATE UNIVERSITY
UNIVERSITY OF NEVADA
NEW MEXICO STATE UNIVERSITY
OREGON STATE UNIVERSITY
UNIVERSITY OF OREGON
OSAKA UNIVERSITY

UNIVERSITY OF BRITISH COLUMBIA CALIFORNIA INSTITUTE OF TECHNOLOGY UNIVERSITY OF CALIFORNIA MONTANA STATE UNIVERSITY NEW MEXICO STATE UNIVERSITY UNIVERSITY OF OREGON OSAKA UNIVERSITY
UNIVERSITY OF SOUTHERN CALIFORNIA STANFORD UNIVERSITY UNIVERSITY OF TOKYO UNIVERSITY OF UTAH WASHINGTON STATE UNIVERSITY UNIVERSITY OF WASHINGTON AMERICAN MATHEMATICAL SOCIETY NAVAL WEAPONS CENTER

* C. R. DePrima California Institute of Technology, Pasadena, CA 91109, will replace J. Dugundji until August 1974. 


\section{Pacific Journal of Mathematics}

\section{Vol. 51, No. $1 \quad$ November, 1974}

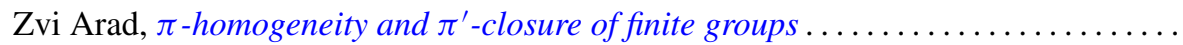

Ivan Baggs, A connected Hausdorff space which is not contained in a maximal

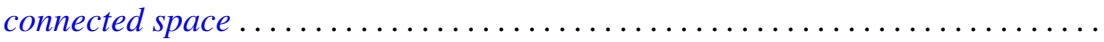

Eric Bedford, The Dirichlet problem for some overdetermined systems on the unit ball in $C^{n}$

R. H. Bing, Woodrow Wilson Bledsoe and R. Daniel Mauldin, Sets generated by

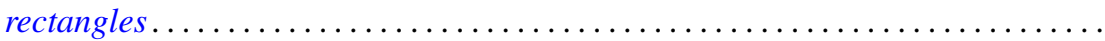

Carlo Cecchini and Alessandro Figà-Talamanca, Projections of uniqueness for

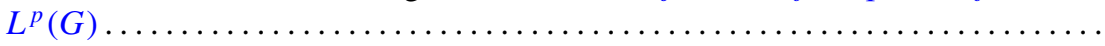

Gokulananda Das and Ram N. Mohapatra, The non absolute Nörlund summability of Fourier series .

Frank Rimi DeMeyer, On separable polynomials over a commutative ring ........ Richard Detmer, Sets which are tame in arcs in $E^{3} \ldots \ldots \ldots \ldots \ldots \ldots \ldots \ldots$

William Erb Dietrich, Ideals in convolution algebras on Abelian groups ..........

Bryce L. Elkins, A Galois theory for linear topological rings .................

William Alan Feldman, A characterization of the topology of compact convergence on $C(X)$.

Hillel Halkin Gershenson, A problem in compact Lie groups and framed cobordism

Samuel R. Gordon, Associators in simple algebras.

Marvin J. Greenberg, Strictly local solutions of Diophantine equations

Jon Craig Helton, Product integrals and inverses in normed rings . . . . . . . . . . . .

Domingo Antonio Herrero, Inner functions under uniform topology . . .

Jerry Alan Johnson, Lipschitz spaces .

Marvin Stanford Keener, Oscillatory solutions and multi-point boundary value

functions for certain nth-order linear ordinary differential equations.

John Cronan Kieffer, A simple proof of the Moy-Perez generalization of the

Shannon-McMillan theorem .......................

Joong Ho Kim, Power invariant rings

Gangaram S. Ladde and V. Lakshmikantham, On flow-invariant sets .

Roger T. Lewis, Oscillation and nonoscillation criteria for some self-adjoint even

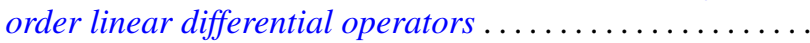

Jürg Thomas Marti, On the existence of support points of solid convex sets ..

John Rowlay Martin, Determining knot types from diagrams of knots . .

James Jerome Metzger, Local ideals in a topological algebra of entire functions

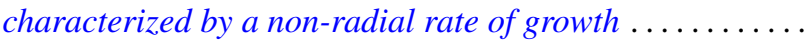

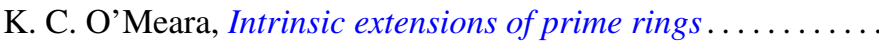

Stanley Poreda, A note on the continuity of best polynomial approximations ..

Robert John Sacker, Asymptotic approach to periodic orbits and local prolongations

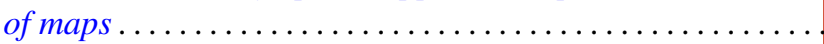

Eric Peter Smith, The Garabedian function of an arbitrary compact set . .

Arne Stray, Pointwise bounded approximation by functions satisfying a side condition

John St. Clair Werth, Jr., Maximal pure subgroups of torsion complete abelian

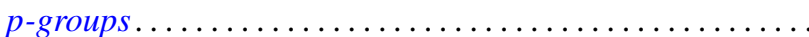

